\title{
An integrated pan-European research infrastructure for validating smart grid systems
}

\author{
T. I. Strasser OVE®, F. Pröstl Andrén, E. Widl@, G. Lauss, E. C. W. De Jong, M. Calin, M. Sosnina, A. Khavari, \\ J. E. Rodriguez, P. Kotsampopoulos, M. Blank, C. Steinbrink, K. Mäki, A. Kulmala, A. van der Meer(D, R. Bhandia,

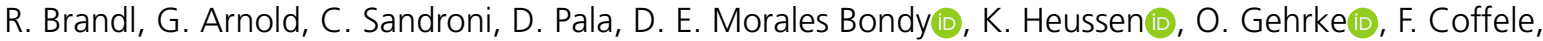 \\ Q.-T. Tran, E. Rikos, V. H. Nguyen (1), I. Orue, M. Z. Degefa(D, S. Manikas
}

A driving force for the realization of a sustainable energy supply in Europe is the integration of distributed, renewable energy resources. Due to their dynamic and stochastic generation behaviour, utilities and network operators are confronted with a more complex operation of the underlying distribution grids. Additionally, due to the higher flexibility on the consumer side through partly controllable loads, ongoing changes of regulatory rules, technology developments, and the liberalization of energy markets, the system's operation needs adaptation. Sophisticated design approaches together with proper operational concepts and intelligent automation provide the basis to turn the existing power system into an intelligent entity, a so-called smart grid. While reaping the benefits that come along with those intelligent behaviours, it is expected that the system-level testing will play a significantly larger role in the development of future solutions and technologies. Proper validation approaches, concepts, and corresponding tools are partly missing until now. This paper addresses these issues by discussing the progress in the integrated Pan-European research infrastructure project ERIGrid where proper validation methods and tools are currently being developed for validating smart grid systems and solutions.

Keywords: smart grids; simulation; hardware-in-the-loop; testing; research infrastructure; education; training

\section{Introduction}

A driving force for the realization of a sustainable energy supply in Europe is the integration of distributed, renewable energy resources $[1,2]$. Due to their dynamic and stochastic generation behaviour, utilities and network operators are confronted with a more complex operation of the underlying distribution grids. Additionally, due to the higher flexibility on the consumer side through partly controllable loads, ongoing changes of regulatory rules, technology developments, and the liberalization of energy markets as outlined in Fig. 1, the system's operation needs adaptation. Sophisticated design approaches together with proper operational concepts and intelligent automation provide the basis to turn the existing power system into a cyber-physical energy system-the smart grid $[3,4]$.

\subsection{Smart grids-towards a higher digitalization} of distribution networks

Whereas transmission systems are already well equipped with sophisticated measurement equipment and are centrally operated, the integration of renewable generators occurs mainly at the level of distribution networks, which used to be operated in a passive way until now. With smaller units at this grid level, the numbers of subsystems and devices to be monitored and controlled steadily increases. While the application of modern scalable Information and Communication Technology (ICT) facilitates this integration, it also creates further coupling of engineering domains where little mutual interdependencies existed before. Challenged by this development, new methodologies and practices must be developed. Viewing the electric energy infrastructure in its entirety as a cyber-physical, critical infrastructure, such new methodologies and practices will have to ensure that the classical high-reliability, real-time operation, and regulatory requirements can be met also in the future. [1-5].
1.2 Needs for integrated and holistic power systems testing The observed increase of complexity thus manifests in increased coupling across domains, such as electricity, automation/control, and ICT, but also in scale and heterogeneity. The effects of this are not trivial to anticipate, nor to summarize: operational aspects become a concern, as failures may propagate across increasingly interdependent automation systems; energy management and coordination may become more challenging. Another challenge with complexity

Paper submitted for the CIGRE Session 2018, SC C6, Paris, France, August 26-31, 2018

Strasser, Thomas I., AIT Austrian Institute of Technology GmbH, Center for Energy, Giefinggasse 2, 1210 Vienna, Austria (E-mail: thomas.strasser@ait.ac.at); Pröstl Andrén, F., AlT Austrian Institute of Technology GmbH, Vienna, Austria; Widl, E., AlT Austrian Institute of Technology GmbH, Vienna, Austria; Lauss, G., AlT Austrian Institute of Technology GmbH, Vienna, Austria; Jong, E. C. W., DNV GL, Arnhem, The Netherlands; Calin, M., DERlab e.V., Kassel, Germany; Sosnina, M., DERlab e.V., Kassel, Germany; Khavari, A., DERlab e.V., Kassel, Germany; Rodriguez, J. E., TECNALIA Research \& Innovation, Derio-Bizkaia, Spain; Kotsampopoulos, P., National Technical University of Athens, Athens, Greece; Blank, M., OFFIS e.V., Oldenburg, Germany; Steinbrink, C., OFFIS e.V., Oldenburg, Germany; Mäki, K., VTT Technical Research Center of Finland, Espoo, Finland; Kulmala, A., VTT Technical Research Center of Finland, Espoo, Finland; Meer, A., Delft University of Technology, Delft, The Netherlands; Bhandia, R., Delft University of Technology, Delft, The Netherlands; Brandl, R., Fraunhofer IEE, Kassel, Germany; Arnold, G., Fraunhofer IEE, Kassel, Germany; Sandroni, C., Ricerca sul Sistema Energetico, Milano, Italy; Pala, D., Ricerca sul Sistema Energetico, Milano, Italy; Morales Bondy, D. E., Technical University of Denmark, Kongens Lyngby, Denmark; Heussen, K., Technical University of Denmark, Kongens Lyngby, Denmark; Gehrke, 0., Technical University of Denmark, Kongens Lyngby, Denmark; Coffele, F., University of Strathclyde, Glasgow, UK; Tran, Q.-T., CEA-INES, Le Bourget du Lac Cedex, France; Rikos, E., CRES, Athens, Greece; Nguyen, V. H., University Grenoble Alpes, G2Elab, Grenoble cedex 1, France; Orue, I., Ormazabal Corporate Technology, Amorebieta, Spain; Degefa, M. Z., SINTEF Energy Research, Trondheim, Norway; Manikas, S., Hellenic Electricity Distribution Network Operator, Athens, Greece 


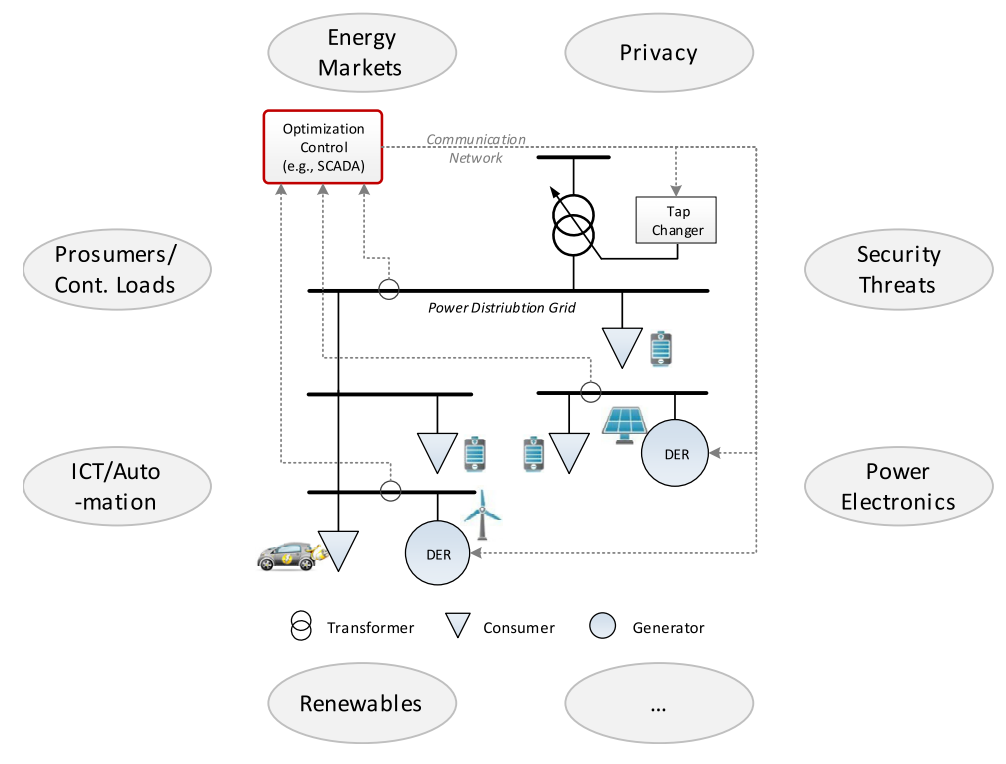

Fig. 1. Influencing factors of smart grid systems

is the ability to conceive, design, and develop critical infrastructure systems that depend on such cross-disciplinary competences.

Before deployment in an operational environment, smart grid solutions and products have to be validated and tested. Industry and researchers have recognized this challenge and according to the 2015 annual report of the Joint Research Center (JRC) of the European Commission (EC), there were 459 projects and demonstrative smart grid platforms in Europe with an overall investment of around $€ 3.15$ billion, in the period from 2002 to 2014 [6]. The growing number of smart grid projects around the world has led to a significant portfolio of demonstrators and advanced ICT networking features.

Testing is an integral part of the development process. The status quo for power systems testing is to focus on a particular Deviceunder-Test, meanwhile simplifying the behaviour of other components to electrical equivalents. This traditional decoupling raises a question of the global behaviours of the integrated system. A combination of different technologies across domains requires that communication among specialists is established and founded on the interconnection of different disciplines during the development process. The heterogeneity of smart grid domains and technologies, notably the interactions between the various technologies, conflicts with the traditional approach: test labs often specialise in a certain domain and can hence only test components for a particular subsystem. So far, testing approaches which combine ICT/automation with electricity domains have had a main focus on individual components [7]. However, in order to support the different stages of the overall development process for smart grid solutions, tests are needed to evaluate the integration on a system level, addressing all relevant test domains $[8,9]$. Proposed alternative testing approaches include virtual (simulation) or semi-virtual (hardware-in-the-loop) experiments that cover multiple domains. For these new approaches, questions arise as to whether the test results can be considered valid enough to draw firm conclusions for a real-world deployment of the tested systems.

\subsection{Contribution}

This paper addresses the above outlined shortcomings on systemlevel testing by discussing the progress of the integrated pan-
European Research Infrastructure (RI) project ERIGrid, where proper validation methods and tools are currently being developed for validating smart grid systems and technologies. Whereas the overall ERIGrid approach and corresponding research directions have already been outlined in a previous paper [10], this work mainly focuses on the achievements and activities related to the definition of a system-level validation process as well as on simulation and lab-based assessment methods. Additionally, the concept of an online coupling of various labs is discussed. A further focus of ERIGrid is on the development of proper training and education material which improves the multi-disciplinary understanding of researchers and engineers which is highly important in the area of smart grids.

The rest of this paper is organized as follows: Sect. 2 provides a brief overview of the overall ERIGrid approach whereas the status of the research and development progress is discussed in Sect. 3. Educational and training aspects are covered in Sect. 4. This paper is concluded with the main findings and an outlook about the planned future work in Sect. 5.

\section{The ERIGrid integrated research infrastructure}

In order to overcome the lack in power system validation and testing as outlined above, eighteen research institutions from eleven European countries are joining their forces and cooperating within the European-funded RI project called "ERIGrid" " [10] which is introduced below.

\subsection{Main goals}

The project aims to address the challenges raised above by developing a holistic, cyber-physical systems-oriented approach for testing smart grid systems. This integrated European smart grid RI targets the following points:

- Creation of a single point of reference promoting research, technology development, and innovation on all aspects of smart grid systems validation,

${ }^{1}$ European Research Infrastructure supporting Smart Grid Systems Technology Development, Validation and Roll Out. 


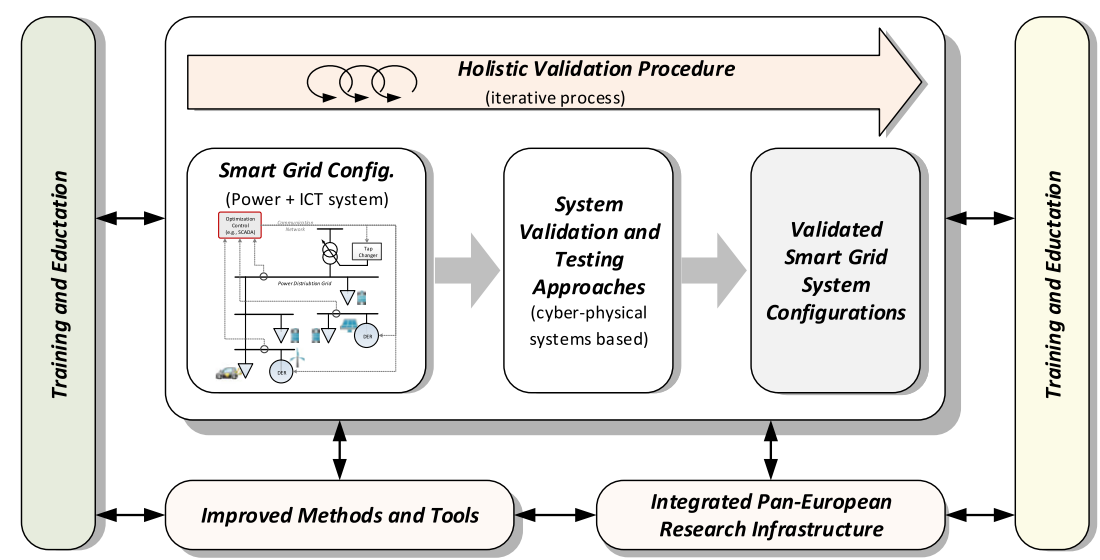

Fig. 2. Overview of the ERIGrid approach (adopted from [10])

- Development of a coordinated and integrated approach using the partners' expertise and infrastructures more effectively, adding value to research projects, and promoting European leadership in smart grid systems,

- Facilitating a wider sharing of knowledge, tools, and techniques across fields and between academia and industry across Europe, and

- Accelerating pre-normative research and promoting the rapid transfer of research results into industrial-related standards to support future smart grids development, validation and roll out.

\subsection{Concept and approach}

In order to realize the above introduced project goals the following main research and development activities have been identified for the ERIGrid project:

- Development of a formalized, holistic validation procedure for testing smart grid systems and corresponding configurations,

- Improvement of simulation and lab-based testing methods supporting the validation activities, and

- The provision of a corresponding and integrated pan-European RI based on the partner's laboratories.

Additionally, training and education concepts are also being developed to support the overall research activities. An interesting point in the ERIGrid approach is to provide free access to the integrated RI (i.e., partner's smart grid laboratories) and the corresponding methods and tools for external user groups from industry and academia as outlined in Fig. 2.

The holistic testing methodology should facilitate conducting tests and experiments representative of integrated smart grid systems by testing and experimentation across distributed Rls, which might not necessarily be functionally interconnected.

\section{Research directions and technology development}

In the following sections the ERIGrid research directions and the status of the corresponding technology development are discussed.

\subsection{Holistic testing of smart grid configurations}

Smart grid solutions have become complex and multidisciplinary. With the further integration of automation, control, ICT and other energy systems new test processes must be defined. A method for framing a holistic approach is being developed in ERIGrid in order to capture this complexity, which aims at enabling the testing of new solutions within their relevant operational context. A holistic testing method ensures a clear vocabulary for smart grid testing across engineering disciplines and a common understanding of how to describe a testing approach that addresses a system-relevant perspective.

The holistic testing vision outlined for ERIGrid is widening the scope of conventional testing by addressing the following points:

- Requirements associated with multiple domains are viewed as part of single test cases,

- Systematic and integrated testing strategy for systems, components and their integration,

- The hybridization of methods applicable to distinct formal representation frameworks (i.e. ICT, discrete and logic oriented testing, vs. physical continuous models and uncertainty),

- The formal integration of several independent tests into a common framework, and

- Technical integration of different means of testing, such as realtime simulation.

Based on these requirements a corresponding holistic testing procedure can be derived, which is outlined in Fig. 3 [11]. The starting point is the specification of a holist test cases, which is usually derived from scenarios and corresponding system configurations (i.e., Step 1). In an independent, parallel step the involved RI(s) (i.e., labs) are profiled regarding their validation capabilities (i.e., Step 2). Afterwards a mapping of the planned test to a corresponding $\mathrm{RI}$ is carried out. A division of the test into sub-tests is also possible if the test cannot be performed in one RI (i.e., Step 3). In Step 4 the concrete design and setup of an experiment is done after the test(s) are defined and the RI is specified. This step is followed by the execution of the experiments (i.e., Step 5), a potential refinement of it if necessary (i.e., Step 6) and the combination of all sub tests into a joint test result (Step 7).

The core elements of this testing approach cover the description of test cases, the test as well as the experiment specification. The (holistic) test case, which describes the test objective in context of a specific smart grid solution, is refined into test specifications that can be split among different Rls, yet still maintaining the overall test problem. Finally, these RIs must then transform these test specifications into experiment specifications that can be carried out at the individual RI. The practical use of the approach is supported by concise definitions, template forms with guiding text, graphical templates as well as exemplary applications.

To support a multi-domain approach, a domain-independent system configuration description method has been defined. This 


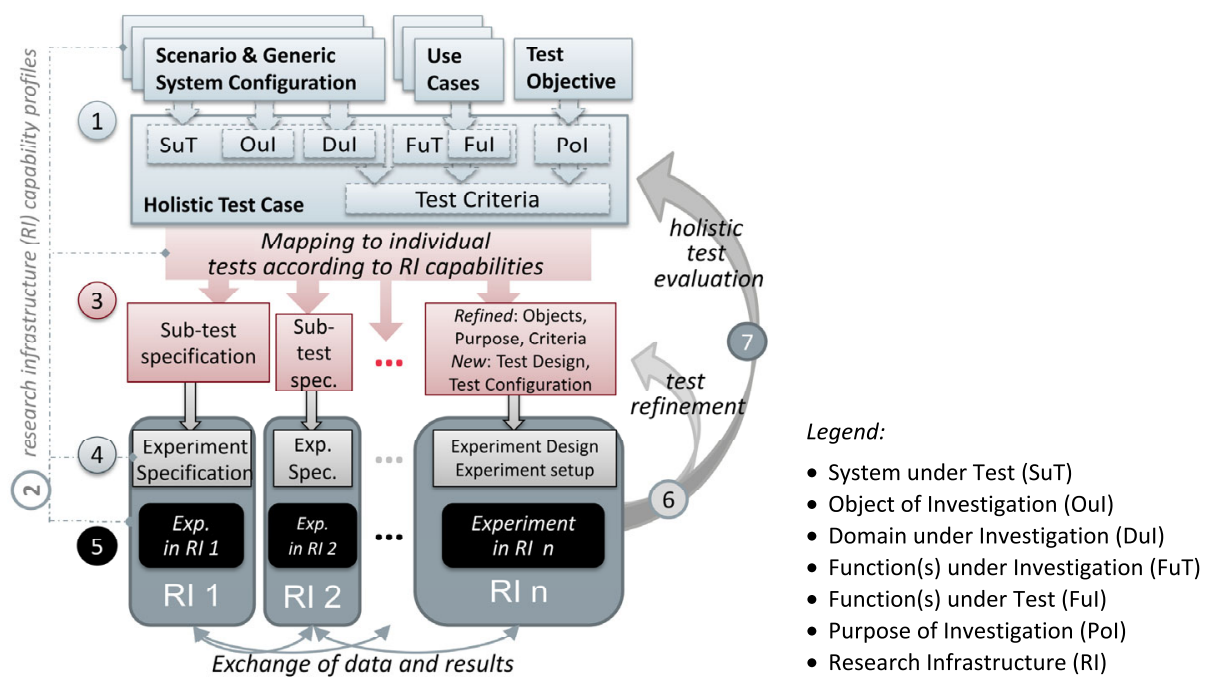

Fig. 3. Proposed holistic testing procedure for smart grid systems [11]

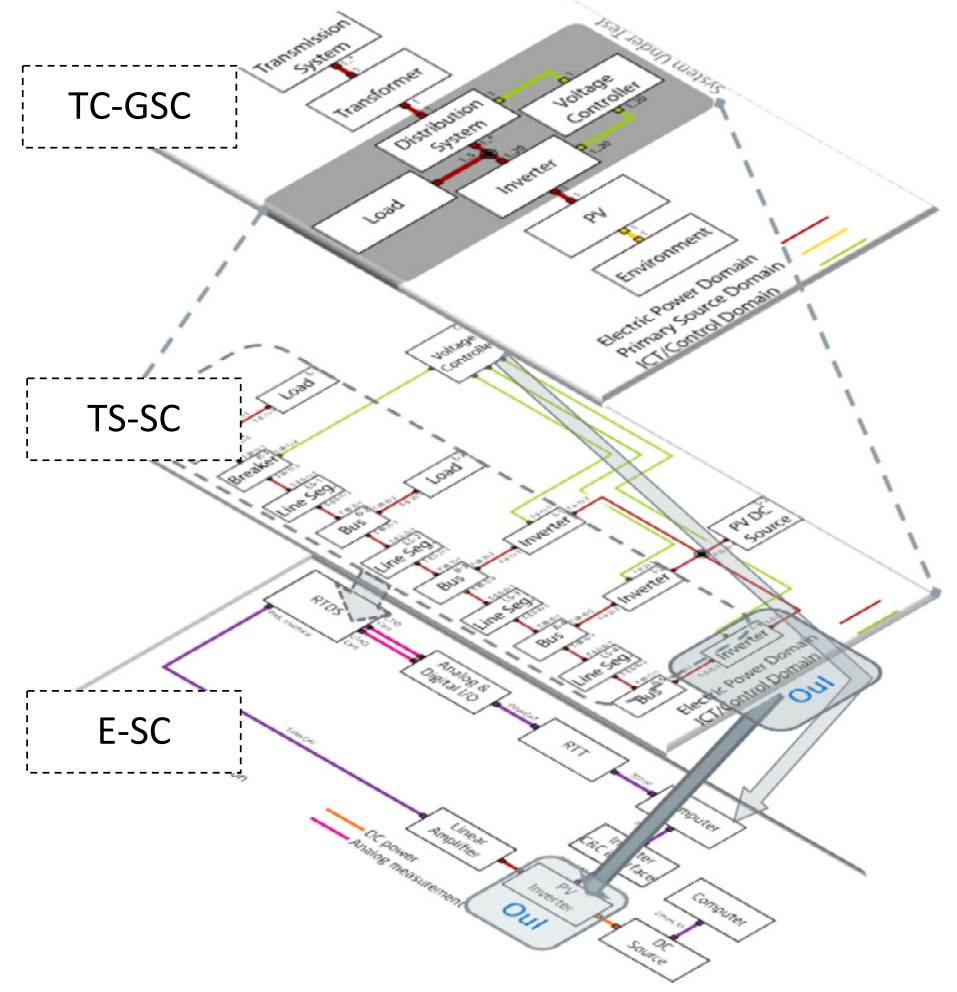

Fig. 4. Intuitive layering of the TC-GSC, TS-SC and E-SC for the same test description

method can be applied to system configurations in several contexts. For each test description template, a related system configuration type is defined: (i) Test Case Generic System Configuration (TC-GSC), (ii) Test Case Specific System Configuration (TS-SC), and (iii) Experiment System Configuration (E-SC). While the GSCS define a contextual information model, a TC-SC defines a specific instance of a system configuration. A visual representation of how the three test case specification layers in terms of associated system configurations (i.e., TC-GSC, TS-SC, and E-SC) connect, is provided in Fig. 4. In this exemplary case, an inverter connected to a distribution system is controlled by a centralized voltage controller. To test an inverter-here the Object under Investigation (Oul)— in closed loop with the voltage controller, an operational context must be identified, called the System under Test (SuT). In the TCGSC, the SuT generic elements and context are identified, which are then refined to a concrete test system in the TS-SC. The TS-SC elements are then mapped into a laboratory context in the E-SC. In this case only the Oul (the inverter) is found as a physical component, and the remaining components for the TS-SC are emulated. 
Addressing the requirements of a testing process, the proposed methodology attempts to strike a balance between formal definitions, existing concepts within standards, and the practical use and understanding of tests. The test case, test- and experiment specifications include the notions of test criteria and parameters, which support the incremental definition of test factors to facilitate the application of the analytical and statistical methods for experiment design and evaluation. Given the test objective, these methods will be used to analytically compose the appropriate test design as well as to enable uncertainty quantification. This will facilitate the exchange and integration of partial test results across Rls and experiments.

ERIGrid's approach on holistic testing may be considered as a vision of a pre-standardised process and methodology implementing the testing of a system that includes multi-domain aspects (e.g., by addressing power, automation/control, and ICT). This vision can be extended to the mutualisation of resources of multiple partners to conduct parallel, sequential and integrated tests according to formalized RI profiles and mapping procedures.

\subsection{Improved assessment methods}

As outlined in the introduction, future power systems will contain a heterogeneous agglomeration of different multi-domain technologies (e.g., power, automation, control, and ICT), which need corresponding assessment methods and tools. The focus of the research activities in ERIGrid are on the further development of simulation and laboratory-based approaches, which allow also a flexible coupling of them in a Hardware-in-the-Loop (HIL) manner [9, 10, 12].

\subsubsection{Simulation-based tools}

As discussed in [12] simulation methods and corresponding tool frameworks are highly important in the engineering and validation of smart grid systems. The focus in ERIGrid is on the further development of proper simulation approaches, especially on the coupling of various tools from different domains (i.e., power system, automation and control, ICT). However, the coupling of different simulation packages leads to a number of serious challenges: different solvers have to interoperate, various numerical phenomena are not well understood, and fundamentally different models must exchange dynamic state information. An emerging industry standard that touches some of these aspects is the Functional Mockup Interface (FMI) [12]. It allows for coupling simulation packages and to encapsulate models. The individual framework components can be tested individually, due to its modular architecture and clean interfaces. However, FMI usually needs a kind of simulation manager for coordinating the different simulation environments. The approach in ERIGrid is to use the open source framework mosaik which provides the corresponding functions and services.

A further focus is on the development of proper component libraries. Existing simulators are usually not capable to cover and describe modern smart grid technology. Certain extensions and modifications are necessary in order to cover power system, automation, and ICT issues. All the developed simulation models must comply with the philosophy of FMI for model exchange in order to fit into the overarching simulation framework.

Figure 5 shows a schematic view of the conceptual co-simulation setup, which is currently being developed and tested. The overall system configuration will be split in separate parts, each represented by an individual Functional Mock-up Unit (FMU) and connected to mosaik, which orchestrates the co-simulation execution. During the execution, mosaik uses the functionality provided by the open source FMI++ library to interact with the FMUs-both FMI for Co-Simulation (CS) and FMI for Model Exchange (ME) are used-and to integrate the models contained in FMUs for ME.

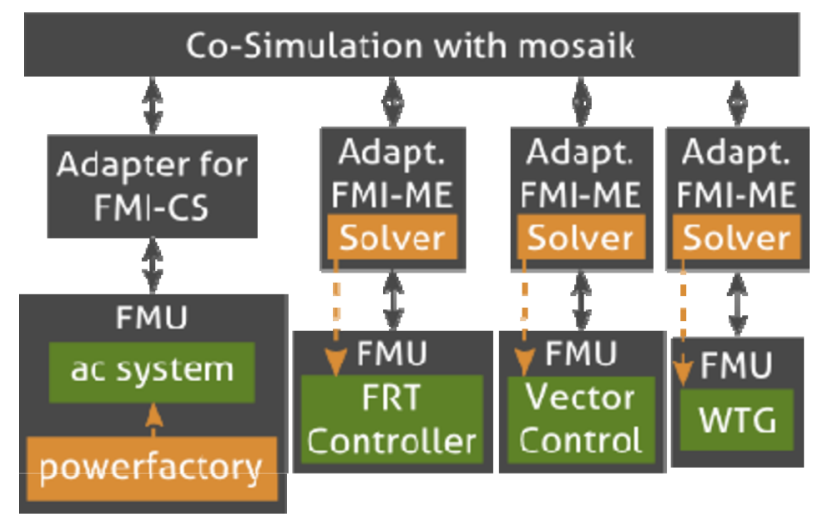

Fig. 5. Schematic of the conceptual co-simulation setup

\subsubsection{Laboratory-based tools}

Also in the focus of the research work is the improvement of labbased testing approaches addressing multi-domain system level issues. This includes the further development of pure lab-based testing approaches integrating power and automation/ICT domains but also the interaction of co-simulation with HIL concepts, as outlined in Fig. 6. With this combination, the strengths of both approaches can be used: multi-domain experiments can be implemented and realized with more realistic behaviours from hardware equipment under a variety of complex environments, co-simulated by appropriate and adapted simulators from the relevant domains.

\subsection{Integrated pan-European research infrastructure}

Smart grid labs are complex infrastructures. They have some unique properties, which distinguish them from other technical installations of comparable complexity. This includes the often-experimental nature of the installation, changing user groups, and evolving configurations. This complicates collaboration between laboratories and presents unique training challenges to resident and visiting users alike. Unlike electrical power grids, whose construction principles have been developed over the course of more than a century and which have been extensively standardised, there is no standard for what constitutes a smart grid laboratory. Consequently, the use of information and communication technology systems, the underlying architectures and the availability of interfaces is subject to large variations between facilities. Therefore, a further research and development activity in ERIGird is the removal of technical and user-related obstacles through standardisation, harmonisation, and documentation.

ERIGrid tackles these issues by introducing a harmonized and detailed description of the available RI capabilities and a coherent and technology-agnostic description of RI control interfaces, supporting the coordination of control software deployment across Rls. Furthermore, if several RIs need to be used in order to realize a testing scenario (as foreseen in the above introduced procedure) a live-coupling of corresponding laboratory components and devices might be required. This leads to the approach called "Joint Research Facility for Smart Energy Networks with Distributed Energy Resources (JaNDER)" [13]. Its main purpose is to integrate all RIs of all ERIGrid partners with secure and interoperable online signal exchange and high-level service provision (e.g., state estimation), providing more opportunities for joint validation of smart grid systems. To facilitate the validation for all partners using joint laboratories, an access from a device interface up to a user interface is needed. The safety and security topics are equally important as the flexible data exchange. 


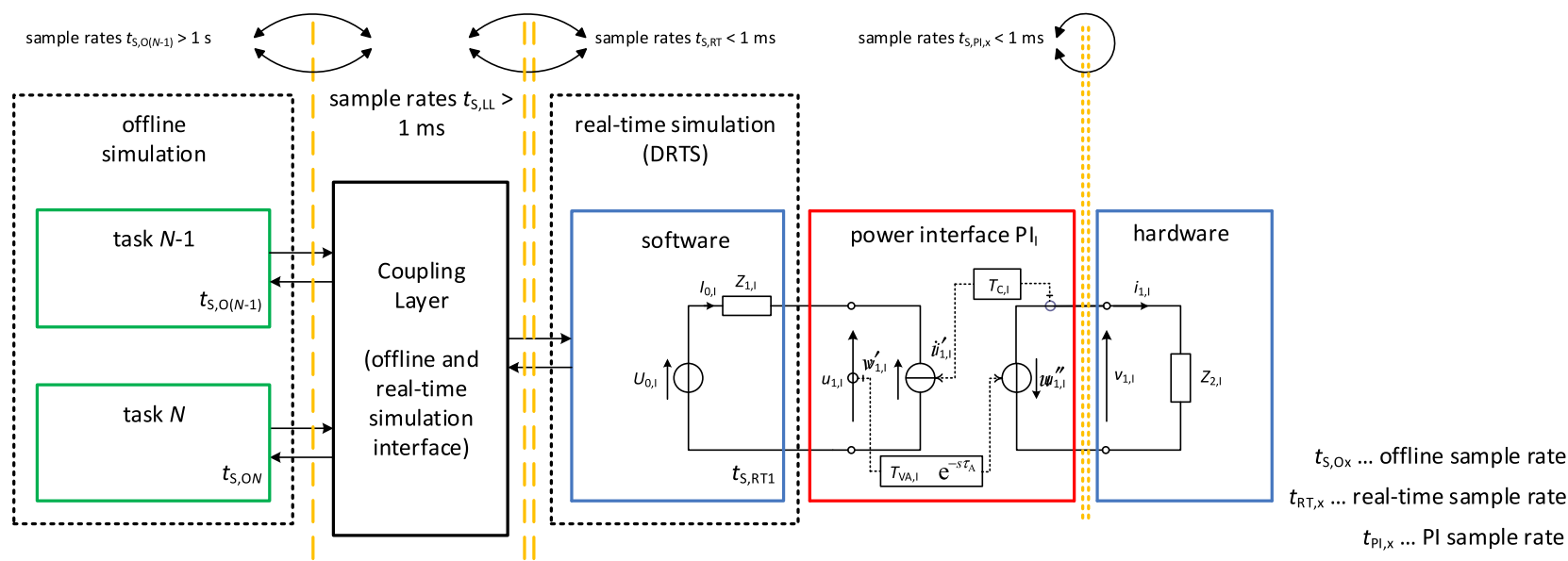

Fig. 6. Combining of HIL and co-simulation methods

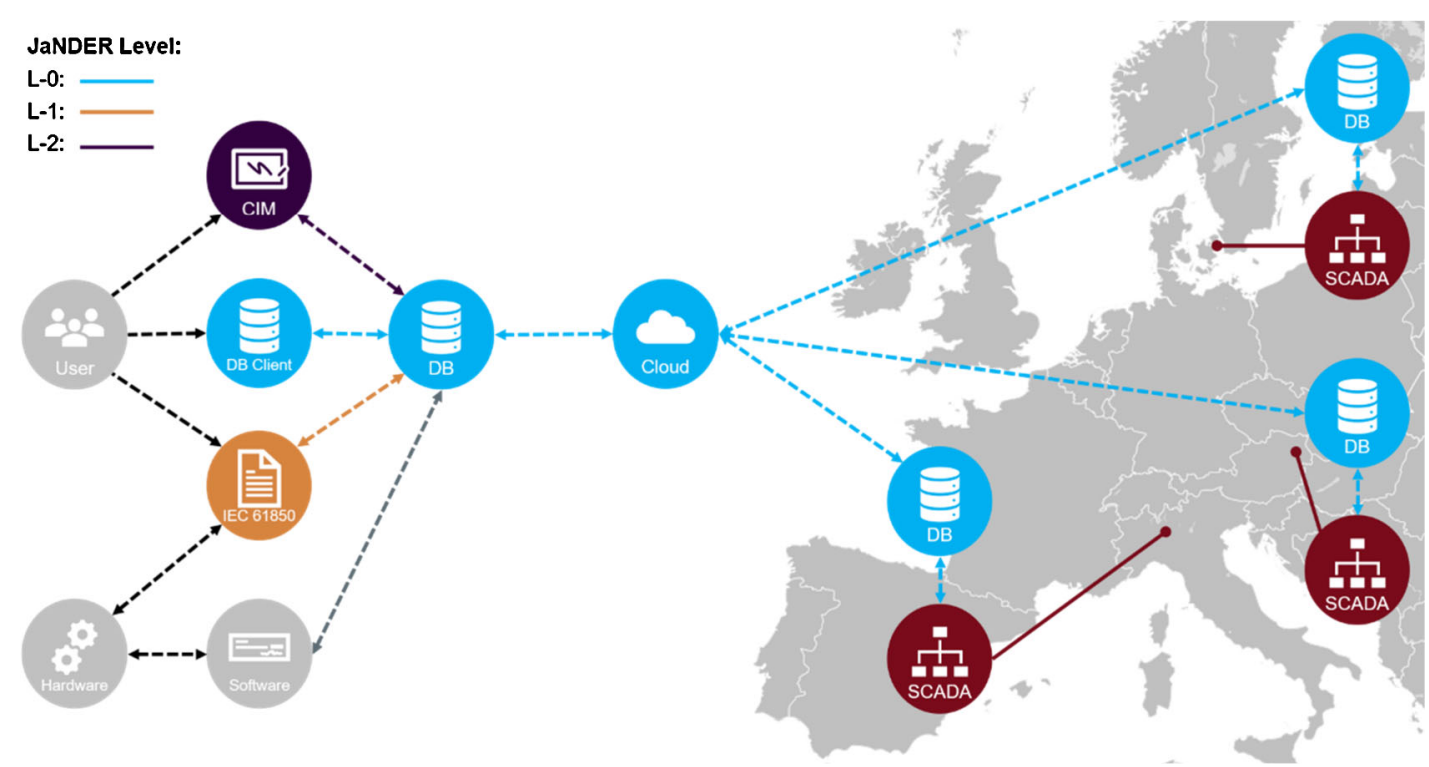

Fig. 7. Online-coupling of smart grid laboratories using the JaNDER approach [13]

As outlined in Fig. 7, the reference points of the JaNDER architecture are local database servers installed at each partner (i.e., connected to the local laboratory automation and SCADA systems). Each of them represents a subscriber, which can choose which data points are available in the corresponding cloud database. The JaNDER implementation of the ERIGrid project provides safe, interoperable, fast and flexible access. Several interfaces are used: (i) Level 0 native database client, (ii) Level 1-IEC 61850, and (iii) Level 2CIM. Additionally, the database servers support more than 40 programming languages for developing new interfaces and direct communications making it relative easy to connect various laboratory components.

The cloud database maintains all shared measurements and control points of all partners' labs forming therefore a virtual, integrated pan-European smart grid RI, which can be used for more complex validation and testing scenarios of smart grid solutions, technologies, and products.

\section{Interdisciplinary education of researchers and engineers}

The complexity of power and energy systems is rising with the integration of renewable and distributed generation, advanced automation and control approaches as well as the usage of sophisticated communication concepts. Therefore, a broad understanding of these topics by researchers and engineers is necessary to develop and validate proper smart grid technologies and solutions. Usually, the classic electrical power engineering education does not sufficiently cover smart grid topics, posing challenges to young researchers and students and also to the industry. Appropriate education and training on modern topics is essential to understand and tackle the challenges and propose new methods, concepts, and corresponding tools as described above [14].

ERIGird is tackling this trend by developing proper education and training material, which is being used in corresponding summer schools, PhD workshops, seminars, webinars, but also laboratorybased experiments. The focus is to educate and train researchers 
and engineers on the multi-disciplinary aspects by understanding the following main topics [14]:

- Physical behaviour of smart grid systems and its connected subsystems and components,

- Automation and control systems,

- Communication networks, and

- Advanced control, optimization, and data analytics.

An interesting example for such a training activity emerged out of the further improvement of HIL-based methods. HIL simulations have been used to show how advanced functions of smart inverters can be used to contribute to power quality improvements. Therefore, in a real-time simulation system the power grid is being modelled and simulated and connected with a real residential PV inverter system. Besides showing the normal operation of the inverter-based system, also emergency situations can be quite easily emulated and correct contingency actions can be trained without any risk of damage in case of a false reaction. Further details about the corresponding curricula are described in [15]

\section{Conclusions}

The expected large-scale roll out of smart grid products and solutions during the next few years requires a multi-disciplinary understanding of several domains. The validation and testing of such complex solutions gets more important as in the past and there is a clear shift from component-level to system-level testing. An integrated, cyber-physical systems-based, multi-domain approach for a holistic testing of smart grid solutions is currently still missing which is addressed by the ERIGrid approach. Four main research priorities have been identified in this project to tackle the shortcomings in today's validation and testing of power systems and corresponding components. The research focus is put onto the development of a holistic validation procedure and the improvement of simulation-based methods, hardware-in-the-loop approaches, and lab-based testing, which can be combined in a flexible manner. The integration and online connection of the partners' labs is also a challenging research and development task in ERIGrid. All these activities need to be supported by the training of researchers and power system professionals. Proper curricula and education material is currently in development.

Summarizing, with the above described integrated pan-European RI approach in ERIGrid the following improved services are being available to the partners but also to external user groups:

- Structured approach for defining a holistic description of validation and testing needs together with corresponding experiment descriptions,

- Improved co-simulation based approach with corresponding FMIbased model libraries addressing the multi-domain and cyberphysical character of smart grid configurations,

- Improved HIL-based concepts analysing system-integration aspects of smart grid components,

- Possibility to couple co-simulation with lab experiments addressing system-integration aspects,

- Evaluation of different ICT and automation architectures, control concepts,

- Support for rapid-prototyping of components and analysing their behaviour in power systems, and
- Support for evaluating different smart grid system configurations.

Future work will mainly cover further improvement of the validation procedure and applying it to selected smart grid scenarios using the improved assessment methods as well as the online-coupling of labs. A large effort will be also put on the education and training of researchers and engineers as well as the provision of the improved $\mathrm{RI}$ capabilities and services to external user groups.

\section{Acknowledgements}

This work is supported by the European Community's Horizon 2020 Program (H2020/2014-2020) under project "ERIGrid" (Grant Agreement No. 654113). Further information is available at the corresponding website www.erigrid.eu.

Open Access This article is distributed under the terms of the Creative Commons Attribution 4.0 International License (http://creativecommons.org/ licenses/by/4.0/), which permits unrestricted use, distribution, and reproduction in any medium, provided you give appropriate credit to the original author(s) and the source, provide a link to the Creative Commons license, and indicate if changes were made.

\section{References}

1. International Energy Agency (IEA) (2011): Technology roadmap: smart grids. Paris, France.

2. European Commission (2016): EU reference scenario 2016. Energy, transport and GHG emissions trends to 2050. Brussels, Belgium.

3. Farhangi, H. (2010): The path of the smart grid. IEEE Power Energy Mag., 8(1), 18-28.

4. Liserre, M., Sauter, T., Hung, J. (2010): Future energy systems: integrating renewable energy sources into the smart power grid through industrial electronics. IEEE Ind. Electron. Mag., 4(1), 18-37.

5. Ardito, L., Procaccianti, G., Menga, G., Morisio, M. (2013): Smart grid technologies in Europe: an overview. Energies, 6(1), 251-281.

6. Catalin, F. C., Miicea, A., Julija, V., Anna, M., et al. (2014): Smart grid projects outlook 2014. JRC science and policy reports. Brussels: European Commission-Joint Research Centre (JRC).

7. Bründlinger, R., Strasser, T., Lauss, G., Hoke, A., et al. (2015): Lab tests: verifying that smart grid power converters are truly smart. IEEE Power Energy Mag., 13(2), 30-42.

8. International Smart Grid Action Network (ISGAN), International Energy Agency (IEA) (2013): Smart Grid International Research Facility Network (SIRFN). Paris, France.

9. Strasser, T., Andrén, F., Lauss, G., Bründlinger, R., et al. (2016): Towards holistic power distribution system validation and testing - an overview and discussion of different possibilities. In CIGRE session 46, Paris, France, Aug. 21-26.

10. Strasser, T. I., Moyo, C., Bründlinger, R., Lehnhoff, S., et al. (2017): An integrated research infrastructure for validating cyber-physical energy systems. In International Conference on Industrial Applications of Holonic and Multi-Agent Systems, HoloMAS, Lyon, France, Aug. 28-30.

11. Blank, M., Lehnhoff, S., Heussen, K., Bondy, D. E. M., et al. (2016): Towards a foundation for holistic power system validation and testing. In 2016 IEEE 21st international conference on emerging technologies and factory automation, ETFA, Berlin, Germany, Sept. 6-9.

12. Steinbrink, C., Lehnhoff, S., Rohjans, S., Strasser, T. I., et al. (2017): Simulation-based validation of smart grids-status quo and future research trends. In International conference on industrial applications of holonic and multi-agent systems, HoloMAS, Lyon, France, Aug. 28-30, 2017.

13. Otte, M., Pala, D., Sandroni, C., Rohjans, S., Strasser, T. (2018): Multi-laboratory cooperation for validating microgrid and smart distribution system approaches. In CIRED workshop 2018, Ljubljana, Slovenia, June 7-8.

14. Kotsampopoulos, P., Hatziargyriou, N., Strasser, T. I., Moyo, C., et al. (2017): Validating intelligent power and energy systems-a discussion of educational needs. In International conference on industrial applications of holonic and multi-agent systems HoloMAS, Lyon, France, Aug. 28-30.

15. Kotsampopoulos, P., Kleftakis, V., Hatziargyriou, N. (2017): Laboratory education of modern power systems using PHIL simulation. IEEE Trans. Power Syst., 32(5), 3992 4001. 